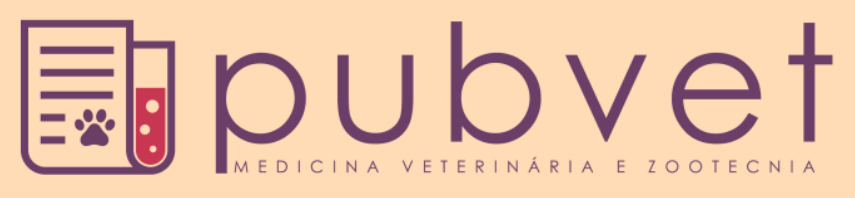

HTTP://DX.DOI.ORG/10.22256/PUBVET.V11N1.30-34

\title{
Aspectos higiênico-sanitários da merenda escolar nas cidades de Bom Jesus e Colônia do Gurgueia - Piauí
}

\author{
Silvia Rayner Rodrigues de Almeida ${ }^{1}$, Janaina de Fatima Saraiva Cardoso ${ }^{2}$, Johnny \\ Iglesias Mendes Araujo ${ }^{3 *}$, Ana Lucia Alves da Silva ${ }^{4}$, Joniel Mendes de Araujo ${ }^{5}$, Ranes \\ Batista da Rocha ${ }^{6}$, Gilvan Rodrigues dos Santos ${ }^{7}$
}

\begin{abstract}
${ }^{1}$ Bacharelada em Medicina veterinária, Universidade Federal do Piauí, Campus professora Cinobelina Elvas, Bom Jesus, Piauí, Brasil. E-mail: Silviarayner@gmail.com

${ }^{2}$ Dra. Biotecnologia/UFPI, Professora do departamento de medicina veterinária/UFPI, Bom Jesus, Piauí, Brasil. E-mail: janainadefatima@hotmail.com

${ }^{3}$ Mestrando em Zootecnia pela Universidade Federal do Piauí, Campus professora Cinobelina Elvas, Departamento de Zootecnia, Bom Jesus, Piauí, Brasil.E-mail: Johnny-iglesias@ hotmail.com

${ }^{4}$ Especialista em Docência e Supervisão Escolar (IESM), Timon, Maranhão, Brasil. E-mail: anaufpi@hotmail.com

${ }^{5}$ Mestrando em Ciências Biológicas, Universidade Federal do Goiás, Campus Samambaia, Departamento de Biologia, Goiânia, Goiás, Brasil. E-mail: niel.ma30@gmail.com

${ }^{6}$ Liceciatura em Ciências Biológicas/UFPI-Campus Prof. ${ }^{a}$ Cinobelina Elvas, Bom Jesus, Piauí, Brasil. E-mail: ranes.ufpi@hotmail.com

${ }^{7}$ Mestrando em Ciências da Educação e Multidisciplinaridade/FACNORTE, E-mail: gr.seduc.pi@gmail.com *Autor para correspondência
\end{abstract}

\begin{abstract}
RESUMO. Esta pesquisa teve como objetivo analisar através da aplicação de um questionário contendo 12 perguntas de ordem sanitária relacionadas as condições higiênico-sanitárias utilizadas no preparo dos alimentos oferecidos nas escolas municipais e estaduais dos municípios de Bom Jesus-PI e Colônia do Gurgueia-PI. Diante dos resultados, identificaram-se os pontos críticos de controle ao qual se deve da ênfase, para que se possa garantir uma merenda escolar de qualidade a milhares de estudantes que passam grande parte da sua vida nas escolas, além de ter a merenda escolar como a principal refeição diária. Dentre as técnicas consideradas incorretas encontram-se também a higienização das mãos dos manipuladores e o uso de detergente ou álcool, além da presença de adornos (brincos, anéis). Concluiu-se que à necessidade da implementação de um esquema de monitoramento sistemático das condições higiênico-sanitárias nas instituições de ensino, além da aplicação de cursos de capacitação dos profissionais que fazem a manipulação dos alimentos a fim de garantir a inocuidade dos alimentos que são fornecidos aos alunos.
\end{abstract}

Palavras chave: Condições higiênico-sanitárias, merenda escolar, segurança alimentar

\section{Aspects in toilet-sanitary school meals in the cities of Bom Jesus and Colônia Gurgueia Piaui-Brazil}

ABSTRACT. This research aimed to analyze through the application of a questionnaire containing 12 questions of health policy related sanitary-hygienic conditions used in the preparation of food offered in municipal and state schools in the municipalities of Bom Jesus-PI and Colônia Gurgueia - PI. Given the results, we identified the critical control points which should be the focus, so that we can secure a school lunch quality to thousands of students who spend much of their lives in schools, in addition to school lunches as the main daily meal. Among the techniques considered incorrect also find themselves hand 
hygiene of food handlers and the use of detergent or alcohol, and the presence of ornaments (earrings, rings). It was concluded that the need to implement a systematic monitoring scheme of sanitary conditions in educational institutions, in addition to implementing training courses for professionals that make the handling of food to ensure food safety that are provided students.

Keywords: sanitary conditions hygienic, school lunch, food safety

\section{Aspectos higiénico sanitarios de la comida escolar en las ciudades de Bom Jesus e Colônia do Gurqueia Piauí -Brasil}

RESUMEN. El presente estudio tuvo como objetivo analizar a través de la aplicación de un cuestionario que contenía 12 preguntas de orden sanitaria relacionadas a la condición higiénica sanitaria utilizada en la preparación de alimentos ofrecidos en las escuelas municipales y estaduales de los municipios de Bom Jesus-PI y Colônia do Gurgueia-PI (Brasil). Frente a estos resultados, se identificaron los puntos críticos de control al cual se debe dar énfasis, para que se pueda garantir una comida escolar de buena calidad a millares de estudiantes que pasan gran parte de su vida en las escuelas, además de tener el almuerzo escolar como la principal comida diaria. Dentro de las técnicas consideradas incorrectas se encontró la mala higienización de las manos de los manipuladores y el uso de detergente o alcohol, además de la presencia de adornos (aretes, anillos). Se concluyó que hay necesidad de implementar un esquema de monitoreo riguroso de las condiciones higiénico sanitarias en las instituciones de enseñanza, además de la aplicación de cursos de capacitación de los profesionales que manipulan los alimentos a fin de garantir la inocuidad de los alimentos que son ofrecidos a los alumnos.

Palabras clave: Condiciones higiénico sanitarias, almuerzo escolar, seguridad alimentaria

\section{Introdução}

No Brasil os programas de alimentação e nutrição em escolas foram criados a partir de 1930 com o objetivo de oferecer refeições a todos os alunos que frequentassem o ensino fundamental em estabelecimentos públicos. Em 1988, a alimentação escolar consagrou-se como um direito constitucional, sendo dever do estado garantir, no mínimo, $15 \%$ das necessidades nutricionais diárias do estudante. A alimentação escolar é um direito do estudante e tem como função à complementação da alimentação familiar (Silva et al., 2003).

A alimentação dentro de padrões higiênicos satisfatórios é uma das condições essenciais para a promoção e a manutenção da saúde, sendo que a deficiência nesse controle é um dos fatores responsáveis pela ocorrência de surtos de origem alimentar (Oliveira et al., 2003).

A qualidade sanitária do alimento depende do controle exercido sobre os perigos químicos, físicos e biológicos, que permeiam todas as etapas da cadeia alimentar, iniciada na produção e finalizada no consumo (Rosa and Carvalho, 2004, Robbs and Campelo, 2002). Os microrganismos que podem causar as doenças veiculadas por alimentos são denominados patogênicos e podem afetar tanto o homem quanto os animais (Figueiredo, 2003).

Os alimentos podem ser contaminados durante todas as etapas, que vão desde a produção até a oferta final, por agentes biológicos constituídos por microrganismos (bactérias, vírus, fungos e parasitas), agentes químicos (desinfetantes, agrotóxicos, detergentes, esmalte e/ ou base) e agentes físicos (cabelo, pedaços de madeira, plástico, palha de aço), sendo os agentes biológicos os principais envolvidos nas doenças transmitidas por alimentos (Serviço Social do Comércio - SESC).

Portanto, objetivou-se com esta pesquisa, realizar um levantamento das condições higiênico-sanitário das cozinhas e dos manipuladores das escolas públicas do município de Bom Jesus-PI e Colônia do Gurgueia - PI pela realização de um questionário socioeducativo com os manipuladores dos alimentos da merenda escolar.

\section{Material e Métodos}

A pesquisa foi desenvolvida em 20 escolas da rede municipal (8 escolas) e estadual (12 escolas) 
pertencente aos municípios de Bom Jesus - PI, e Colônia do Gurgueia - PI. Os municípios são compreendidos pelas coordenadas geográficas: latitude $09^{\circ} 04^{\prime} 28^{\prime \prime}$ sul e a uma longitude $44^{\circ} 21^{\prime}$ $31 "$ oeste, estando a uma altitude de 277 metros e latitude $07^{\circ} 13^{\prime} 52^{\prime \prime}$ sul e a uma longitude $42^{\circ} 10^{\prime}$ 41" oeste, estando a uma altitude de 0 metro, respectivamente. A pesquisa foi realizada durante o período de agosto a setembro de 2013. Foram 40 funcionários (merendeiras) participantes da pesquisa, sendo dois (2) por escolas.

Para avaliar as condições higiênico-sanitárias da cozinha, e dos manipuladores (merendeiras) foram analisados itens em cada estabelecimento de ensino, relacionados às condições das instalações; a higienização das mãos dos manipuladores, cuidados com os utensílios; produção das refeições; e o conhecimento dos manipuladores sobre as boas práticas de fabricação e dos procedimentos operacionais padronizados.

Para a coleta dos dados foram realizadas por meio de um questionário contendo 12 perguntas direcionadas aos manipuladores de alimentos das redes de ensino público fundamental e médio nos municípios de Bom Jesus-PI e Colônia do Gurgueia - PI, como: 1. Ao ser admitido, fez exames médicos e laboratoriais? (Parasitológico, hemograma, culturas): ( ) Sim ( ) Não Qual?; 2. Faz a higienização da cozinha (Chão, Parede, Utensílios) no início e no termino do preparo dos alimentos: ( ) Sim ( ) Não Final? Início e final?; 3. Trabalha sem a existência de feridas e supurações no corpo?: ( ) Sim ( ) Não Local?; 4. Lava cuidadosamente as mãos antes da manipulação de alimentos?: ( ) Sim ( ) Não Detergente? Detergente e Álcool?; 5. Fez ou faz algum tipo de programa de capacitação profissional relacionado à higiene pessoal e à manipulação de alimentos?: ( ) Sim ( ) Não Qual? Quando?: 6. Utiliza uniformes claros, limpos apropriados para a manipulação de alimentos?: ( ) Sim ( ) Não Touca? Touca e Avental?; 7. Mantém unhas cortadas e sem esmalte?: ( ) Sim, ( ) Não Frequência?; 8. Presença de insetos no ambiente?: ( ) Sim ( ) Não Frequência?: 9. Trabalha sem adornos? (Relógios, brincos, pulseiras, anéis, etc.): ( ) Sim ( ) Não Frequência?: 10. Tem consciência que os alimentos podem transmitir doenças e causar até a morte?: ( ) Sim ( ) Não; 11. Conhece as exigências higiênico-sanitárias impostas aos manipuladores de alimentos?: () Sim ( ) Não Conhece? Não Conhece?; 12. Preocupa-se com a qualidade e segurança do alimento que manipula? ( ) Sim ( ) Não Frequência?.

Por meio destes questionários buscaram-se desprender informações sobre saúde, grau de instrução, hábitos higiene corporal e hábitos pessoais dos manipuladores de alimentos nas escolas analisadas.

\section{Resultados e Discussão}

Diante dos resultados, identificaram-se os pontos críticos de controle ao qual se deve da ênfase, para que se possa garantir uma merenda escolar de qualidade a milhares de estudantes que passam grande parte da sua vida nas escolas, além de ter a merenda escolar como a principal refeição diária.

Em relação à higiene dos alimentos dos utensílios utilizados na preparação dos alimentos e dos manipuladores, observou-se uma minoria de amostras dentro dos padrões exigidos pelas boas práticas de fabricação. Dentre as técnicas consideradas incorretas encontram-se também a higienização das mãos dos manipuladores e o uso de detergente ou álcool, além da presença de adornos (brincos, anéis).

Após a análise dos resultados obtidos com o questionário foi verificado que $98 \%$ dos manipuladores lavam as mãos com frequência, e destes, $92 \%$ lavam as mãos com detergente e os outros 6\% com detergente e álcool (Gráfico 1). Estudos têm demonstrado que inúmeros surtos alimentares têm sido frequentemente associados à precária higiene pessoal dos manipuladores. De fato, eles exercem uma função importante na segurança alimentar por poderem introduzir patógenos nos alimentos durante a produção, processamento, distribuição e o preparo. A presença de microrganismo patogênico nas mãos representa grande importância epidemiológica, devido à possibilidade de transferência cruzada para os alimentos (Navarro, 2000).

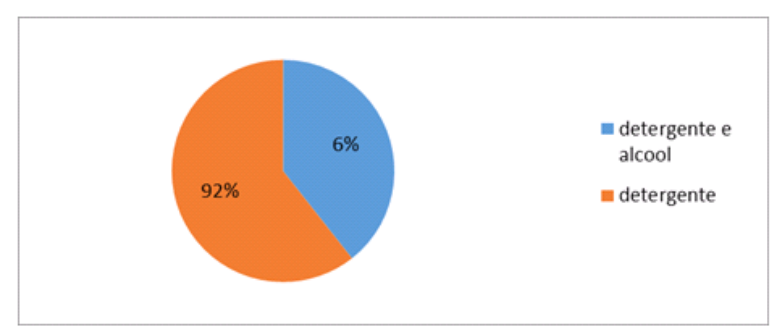

Gráfico 1. Porcentagem de Manipuladores que lavam as mãos somente com detergente ou utilizam detergente e álcool 
De acordo com Silva et al. (2003) os manipuladores de alimentos, são tidos como principais veículos de contaminação dos alimentos, ao manipulá-los de forma inadequada, sendo também um potencial disseminador de bactérias, principalmente devido à ausência de hábitos de higiene pessoal, como a lavagem de mãos, ou quando apresentam ferimentos e infecções na pele.

Figueiredo (2003) recomenda um processo de higienização correta das mãos após manipular qualquer outro objeto antes da manipulação. Conforme o Ministério da saúde (RDC $\mathrm{n}^{\circ}$ 216/2004), para lavar as mãos corretamente devese seguir os seguintes passos: utilizar água corrente para molhar as mãos; esfregar a palma e o dorso das mãos com sabonete, inclusive as unhas e os espaços entre os dedos, por aproximadamente 15 segundos; enxaguar bem com água corrente retirando todo o sabonete; secar com papel toalha ou outro sistema de secagem eficiente e por último, esfregar as mãos com um pouco de antisséptico.

De acordo com os dados no Gráfico 2 foi possível observar que $90 \%$ dos manipuladores utilizam vestimentas limpas e de cor clara ao manipular os alimentos, destes um percentual de $75 \%$ só utiliza touca e avental e $15 \%$ utilizava somente toca. A vestimenta é considerada um ponto crítico para a contaminação, pois o uso de uniforme completo, limpo e de cor clara é essencial para a realização da manipulação de alimentos, devendo ser usado diariamente e exclusivo para a área de manipulação de alimentos e refeições (Souza and Germano, 2004). Para Germano and Germano (2000), a contaminação de alimentos secos pode ocorrer através da água, dos recipientes e utensílios contaminados e dos próprios manipuladores.

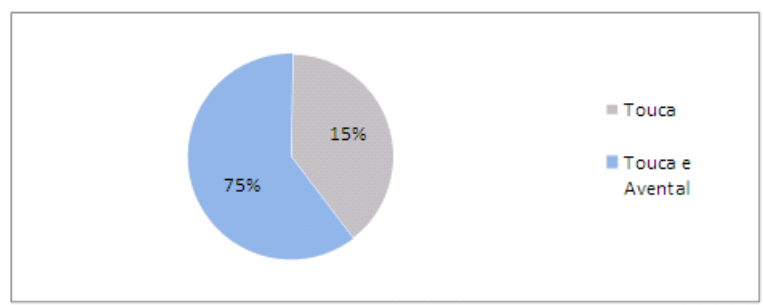

Gráfico 2. Porcentagem de Manipuladores que utilizam touca e avental ou somente touca na manipulação dos alimentos

Verificou-se no Gráfico 3, que $100 \%$ dos manipuladores que fazem a higienização da cozinha e utensílios, destes apenas $75 \%$ realizam essa higienização no início e no final do uso dos mesmos, já os outros $25 \%$ realizam apenas no fim. Como afirmam Ferreira et al. (2004), a limpeza é necessária para proteger os alimentos de modo a não entrarem em contato com os microrganismos, além disto, todas as superfícies em contato com alimentos devem ser limpas e higienizadas antes do uso e depois de cada interrupção, quando elas podem se contaminar. Nos casos de operação contínua de produção, os utensílios e as superfícies dos equipamentos que entram em contato com os alimentos devem ser limpas e higienizadas quando necessário.

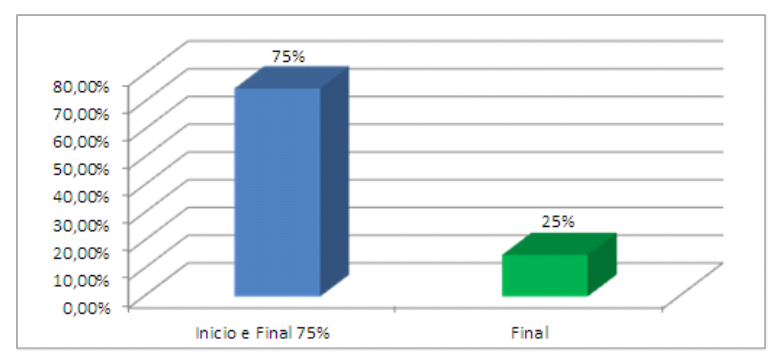

Gráfico 3. Porcentagem de Manipuladores que fazem a higienização da cozinha e utensílios somente no final ou início e fim do preparo dos alimentos.

Ao serem questionados sobre o conhecimento das exigências higiênico-sanitárias e boas práticas de fabricação impostas aos manipuladores $95 \%$ tinham conhecimento sobre o assunto e destes apenas $75 \%$ já haviam participado de uma capacitação sobre manipulação de alimentos, o que é preocupante (Gráfico 4).

Visto que esses cursos são importantes, pois auxiliam na escolha do procedimento a ser adotado durante o preparo de refeições, já que o manipulador de alimentos é um ponto determinante na contaminação dos alimentos, pois estão em contato mais próximo com os alimentos e muitas vezes apresentam atitudes insatisfatórias em relação aos cuidados higiênicos sanitários (Almeida et al., 2002; Nolla and Santos, 2005).

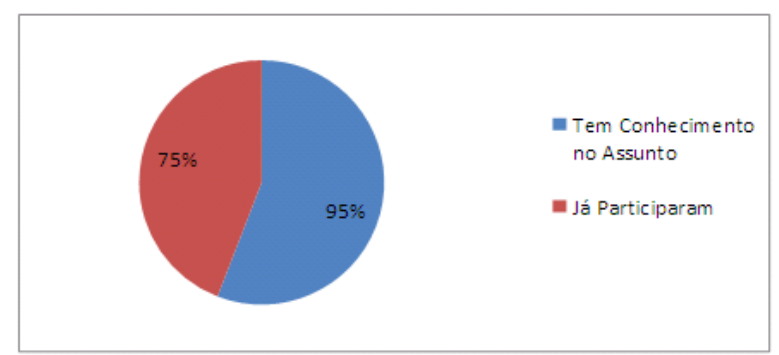

Gráfico 4. Porcentagem de Manipuladores que já participaram ou tem Conhecimento das exigências higiênico-sanitárias e boas práticas de fabricação impostas aos manipuladores. 
Em relação ao estado de saúde dos manipuladores apenas $40 \%$ afirma que fez algum tipo de exame médico ou laboratorial ao ser admitido, A Portaria 6/99 de 10 de março de 1999 rege que trabalhador que manipula ou entra em contato com o alimento não pode ser portador aparente ou inaparente de doenças infecciosas ou parasitárias, e que a periodicidade dos exames médicos e laboratoriais, incluindo o coproparasitológico, devem ser anual (Madeira and Ferrão, 2002). Os manipuladores com sinais de diarreia, febre, faringite, sinusite, devem ser afastados do ambiente de trabalho até a sua recuperação, sendo assim pode se evitar que alunos não venham se contaminarem pelo consumo de alimentos contaminados pelo manuseio, preparo ou pela falta de higiene pessoal dos manipuladores.

\section{Conclusão}

Após análise dos dados obtidos na pesquisa, verificou-se que se faz necessário a implementação de cursos de capacitação continua aos manipuladores, abordando temas ligados a segurança alimentar, nutrição, técnicas de preparo, forma correta de armazenamento dos produtos, controle de pragas, uso de uniformes completos e limpos e a correta higienização das mãos. Pois assim, os microrganismos patogênicos serão mantidos afastados do manipulador e do ambiente de preparo dos alimentos, garantindo sanidade ao consumidor final. Além da melhoria das estruturas e espaços destinados ao preparo dos alimentos, visando à produção de alimentos seguros, que não venham a pôr em risco a saúde dos consumidores.

\section{Referências Bibliográficas}

Almeida, G. D., Jorge, I. M. G., Gabriel, F. H. L., Yajima, R., Silva, E. O. T. R. \& Balian, S. C. 2002. Produção de refeições em creche: recursos para a implementação das boas práticas de higiene e manipulação de alimentos, em busca de qualidade. Higiene Alimentar, 16, 26-29.

Ferreira, C. E. M., Bezerra, L. G. \& Neto, G. V. 2004. Guia para implantação de boas práticas de fabricação (BPF) e do Sistema APPCC. Rio de Janeiro.

Figueiredo, R. M. 2003. As armadilhas de uma cozinha. Manole.
Germano, P. M. \& Germano, M. I. S. 2000. Food Sanitary surveillance as a Health Promotion factor. Mundo saúde, 24, 59-56.

Madeira, M. \& Ferrão, M. E. M. 2002. Alimentos conforme a lei. Editora Manole Ltda.

Navarro, S. H. V. R. 2000. Treinamento para manipuladores de alimentos: enfoque nas técnicas de treinamento exemplificado com a lavagem das mãos. Universidade de São Paulo, São Paulo.

Nolla, A. C \& Santos, G. A. 2005. Relação entre ocorrência de enterro parasitose em manipuladores de alimentos e aspectos epidemiológicos em Florianópolis, Santa Catarina, Brasil. Caderno de Saúde Pública, 641-645.

Oliveira, A. D. M., Gonçalves, M. O., Shinohara, N. K. S. \& Stamford, T. L. M. 2003. Manipuladores de alimentos: um fator de risco. Higiene Alimentar, 17, 12-8.

Robbs, P. G. \& Campelo, J. C. F. 2002. Produção segura na cadeia do leite. Segurança Alimentar na Cadeia do Leite, 1, 51-76.

Rosa, O. O. \& Carvalho, E. P. 2004. Implementação do sistema de análise de perigos e pontos críticos de controle (APPCC) para o controle de qualidade de produtos minimamente processados. Higiene Alimentar, 18, 30-36.

Silva, C., Germano, M. I. S. \& Germano, P. M. L. 2003. Conhecimento dos manipuladores da merenda escolar em escolas da rede estadual de ensino em São Paulo-SP. Higiene Alimentar, $17,46-51$.

Souza, R. R. D., Germano, P. M. L., \& Germano, M. I. S. 2004. Técnica da simulação aplicada ao treinamento de manipuladores de alimentos, como recurso para a segurança alimentar de refeições transportadas. Higiene Alimentar, 18, 21-25.

\section{Article History:}

Received 7 October 2016

Accepted 17 October 2016

Available on line 7 November 2016

License information: This is an open-access article distributed under the terms of the Creative Commons Attribution License 4.0, which permits unrestricted use, distribution, and reproduction in any medium, provided the original work is properly cited. 